\title{
DEVELOPMENT OF A CAPACITIVE MEMS RF POWER SENSOR WITHOUT DISSIPATIVE LOSSES: TOWARDS A NEW PHILOSOPHY OF RF POWER SENSING
}

Luis J. Fernández, Eelke Visser, Javier Sesé, Remco Wiegerink, Henri Jansen, Jaap Flokstra and Miko Elwenspoek

MESA+ Institutc for Nanotcchnology, University of Twentc, P.O. Box 217, 7500AE, Enschede, The Nctherlands

1.j.Fernandez@el.utwente.nl

\begin{abstract}
The development of a novel radio frequency (rf) power sensor is presented based on capacitive micro electromechanical system (MEMS) technology, in which the signal is barely disturbed during the power measurement. Results of the first prototypes and improvements for the second generation of $\mathrm{rf}$ power sensors are given.
\end{abstract}

\section{Introduction}

The high performance of MEMS has been proven and used for the fabrication of gyroscopes, accelerometers and pressure sensors in the past decades. Recently, the development of new components for precise electrical metrology applications based on capacitive MEMS technology has been proposed [1]. Making use of the high stability properties of MEMS, electrical metrology devices like an ac/dc converter, an accurate dc reference and a novel kind of rf power sensor could be realized based on this technology [2].

Apart from its high performance and stability, the relative easy integration with electronics, batch fabrication ability (and thus in potential low fabrication cost) and small size, make MEMS very promising candidates for precise electrical components in the future.

In this paper, the development of an rf power sensor is presented based on capacitive MEMS technology. The if power detection is realized by the movement detection of a grounded suspended membrane placed above of a planar transmission line where the if signal is traveling. The movement detection is done capacitively. Successful results obtained with the first prototypes are shortly presented, and improvements that are being implemented are discussed.

\section{Operation principle}

For a transmission line with a specific characteristic impedance, the power level of an rf signal is completely determined by the rms voltage, $V_{\text {rms. }}$. When a movable grounded membrane is placed above the line where the signal is passing, a shunt capacitor is introduced, and a force between the plates due to the difference in voltage will appear. For frequencies much higher than the mechanical resonance frequency of the movable membrane, this force will be proportional to the power level since the membrane will be only sensitive to the square of the $\mathrm{V}_{\mathrm{rms}}$, and the membrane will be pulled towards the transmission line. Therefore, the power level can be deduced by detecting the movement of the plate.

\section{First prototype}

\section{Design and fabrication.}

The first prototype of the sensor consists of an on both sides clamped grounded membrane or bridge placed above a coplanar waveguide (CPW), where the rf signal is traveling (see figure 1). Sensing electrodes are placed below the movable membrane in order to detect its movement capacitively. The measuring principle is then the following: The rf signal will bend the membrane downwards (as explained before). The displacement is detected by capacitance change measurements between the sensing electrodes and the membrane, and the rf power is then deduced.

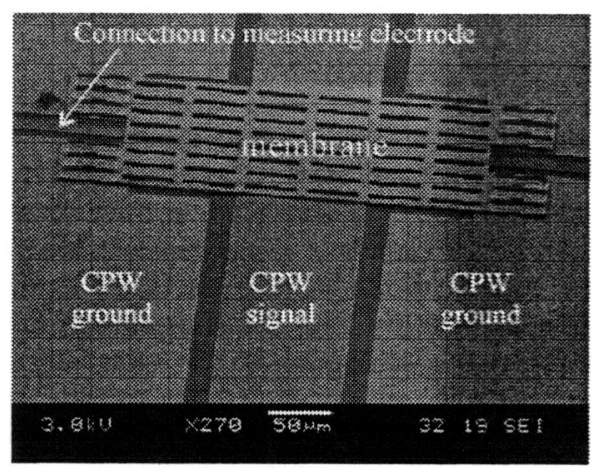

Figure 1: SEM picture of the first prototype (top view)

The fabrication of the sensor was done by surface micromachining. AF45 glass wafer was chosen as substrate material because of its good if properties and aluminum was the metal used for the fabrication of the CPW and the conducting movable plate.

\section{Measurement results.}

S-parameters characterization was realized with a HP8510C Vector Network Analyzer and Cascade Microtech 9000 probe station. The results showed a very good agreement between theory and experimental values [3]. The reflection losses could be theoretically predicted by the capacitance value introduced by the sensor, and the transmission losses were explained by adding the 
expected resistive losses coming from the length of the $\mathrm{CPW}$. In this way, we could conclude that the $\mathrm{S}$ parameters are limited by the capacitance value coming from the membrane, and the length of the CPW.

The capacitance between the membrane and the sensing electrode was measured as a function of the signal power introduced in the CPW, and a linear relation was found proving the operation principle of the measurement. With the first prototype, the sensitivity was enough to measure power levels in the order of milliwatts [3].

\section{Second prototype}

\section{$\underline{\text { S-parameters. }}$}

The goal concerning the reflection losses of the sensor is to achieve $S_{11}$ better than $-26 \mathrm{~dB}$ up to $4 \mathrm{GHz}$. As we have learned from the results of the first prototype, if we want to reach that value, the capacitance introduced from the membrane should not be larger than $80 \mathrm{fF}$, instead of the $180 \mathrm{fF}$ value of the first generation. Concerning the transmission losses, the goal is to get no more losses than $\mathrm{S}_{12}=-0.1 \mathrm{~dB}$. From the results obtained in the first prototype, we can deduce than the length of the $\mathrm{CPW}$ should be not longer than $1 \mathrm{~mm}$. With this new design, we expect to fulfill all our S-parameters specifications with the second generation of rf power sensors.

\section{Temperature dependence.}

Due to the difference in the thermal expansion coefficient between the substrate material $\left(5.4 \cdot 10^{-6} / \mathrm{K}\right)$ and the aluminum used for the fabrication of the membrane (22.6. $10^{-6} / \mathrm{K}$ ), the sensor suffers temperature dependency. When there is a change on the temperature of the surrounding atmosphere, the difference in the expansion of these two materials results in a bending of the membrane. In the new generation of sensors, the springs are created by meander shape supports (see figure 2), which absorb the stress created in the membrane. In this way, temperature independent capacitance measurements are possible. Other materials, which similar expansion coefficient than the glass substrate, like molybdenum $\left(4.5 \cdot 10^{-6} / \mathrm{K}\right)$ are being studied.

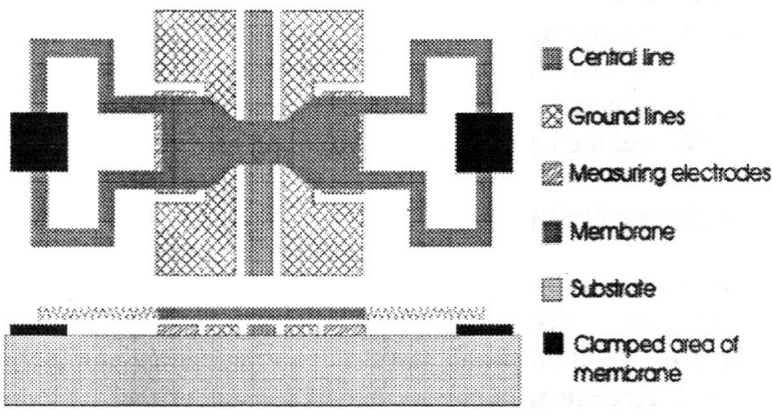

Figure 2: Schematic design (top view and cross section) of the second generation of power sensors

\section{Self heating.}

Self-heating of the sensor has been observed by comparison of the response when dc actuation was used with and without current through the CPW. In this case, the shorter length of the transmission line discussed before is thought to reduce this effect.

\section{Capacitance measurement.}

In the first prototype the membrane is connected to ground. This ends in an increment of the measuring capacitance due to any parasitic capacitance added in parallel to the ground. In the new design, a floating membrane is created, which allows elimination of parasitic capacitance and provides a much more accurate capacitance measurement. We expect to gain a factor of 10 in the power resolution due to this change.

\section{Sensitivity.}

The resolution was also limited by the high spring constant values coming from the two sides supported configuration. With the new meander springs, we expect to reduce the spring constant of the sensor by a factor of 100 , with a subsequent gain in resolution.

\section{Conclusions}

Results of the first generation of $\mathrm{rf}$ power sensors based on a novel principle were reported. Improvements in the design that are being implemented in the second generation of sensors are presented. In the new generation, a power resolution of microwatts and Sparameters of $S_{11}=-26 \mathrm{~dB}$ and $S_{12}=-0.1 \mathrm{~dB}$ are expected. The measurement results of the second generation of $\mathrm{rf}$ power sensor will be presented at the conference.

\section{Acknowledgements}

This work is part of the IST-2000-28261 EMMA programme of the European Commission. We wish to thank Tauno Vähä-Heikkilä, Jukka Kyynäräinen, Aarne Oja, Jussi Varis, Heikki Seppä and Anu Kärkkäinen from VTT for useful discussions.

\section{References}

[1] Seppä H., Kyynäräinen J., Oja A.: "Microelectromechanical systems in electrical metrology", IEEE Trans. Instrum. and Meas., Vol. 50 Issue: 2, pp 440-444, 2001

[2] Tauno Vähä-Heikkilä, Jukka Kyynäräinen, Aarne Oja, Jussi Varis, and Heikki Seppä, "Capacitive MEMS power sensor", MEMS WAVE Workshop, Crete, 2002

[3] L.J. Fernandez, E. Visser, J. Sesé, R.J. Wiegerink, H.V Jansen, J. Flokstra, and M. Elwenspoek, "High frequency power sensor based on MEMS technology," IEEE Sensors Conference, Toronto, October 22-24, 2003. 